1 Molecular therapeutics of pancreatic ductal adenocarcinoma:

2 targeted pathways and the role of cancer stem cells

3

4

5 Andrei-Florian Stoica, Chao-Hui Chang and Siim Pauklin*

6

7 Botnar Research Centre, Nuffield Department of Orthopaedics, Rheumatology and

8 Musculoskeletal Sciences, Old Road, University of Oxford, Oxford OX3 7LD

9

* Correspondence: siim.pauklin@ndorms.ox.ac.uk

10

11 Keywords

12

Pancreatic ductal adenocarcinoma; Cancer therapy; Cancer Stem Cells; Signalling pathways;

13

Tumour stroma; Cancer hallmarks.

14

15

16

17

18

19 
Pancreatic ductal adenocarcinoma (PDAC) is one of the most lethal cancers in human due to late detection and highly metastatic characteristics. With a dismal survival rate of under $8 \%$ and increasing incidence of risk factors including obesity and metabolic syndrome, PDAC is projected to become the second cause of cancer-related death. PDAC cells vary in their tumourigenic capabilities with the presence of a subset of PDAC cells known as pancreatic cancer stem cells (CSCs) which are more resistant to currently used therapeutics. We describe the role of CSCs and tumour stroma in developing therapeutic strategies for PDAC and suggest that developmental plasticity could be considered a hallmark of cancers. We provide an overview of the molecular targets in PDAC treatments including targeted therapies of cellular processes such as proliferation, evasion of growth suppressors, activating metastasis, and metabolic effects. Since PDAC is an inflammation-driven cancer we also revisit therapeutic strategies targeting inflammation, and immunotherapy. Lastly, we suggest that targeting epigenetic mechanisms opens therapeutic routes for heterogeneous cancer cell populations including CSCs. With the accumulation of mechanistic insight, these novel strategies are expected to increase the therapeutic efficiency as personalised individual or combined treatments, and lead to increased survival of PDAC patients. 


\section{Pancreatic ductal adenocarcinoma}

Pancreatic ductal adenocarcinoma (PDAC) is one of the most lethal human malignancies[1]. With a survival rate of $8 \%[1]$, it has the most dismal prognosis of all gastrointestinal tumours[2], and is currently the fourth most common cause of cancer-related mortality in the Western world[3]. Furthermore, with the increasing incidence of risk factors including obesity and metabolic syndrome (see Glossary)[4], PDAC is projected to overtake breast, prostate and colorectal cancers[5] and to become the second cause of cancer-related death in the US by 2030[2]. The ever-increasing number of PDAC cases and the disease's high mortality account for a near-parity between the numbers of new diagnoses of PDAC and the numbers of deaths due to the disease reported in each year[6].

PDAC owes its exceptional lethality to multiple factors. As of 2020 , surgery remains the only curative treatment. The disease often has no early symptoms and presents itself in an advanced stage at diagnosis: only $20 \%$ of newly diagnosed pancreatic cancers are amenable to surgery[7]. $15-20 \%$ of new patients present locally advanced cancer and the remaining $60-65 \%$ present metastatic cancer at diagnosis[8]. Furthermore, the disease's poor response to chemotherapy and radiotherapy results in disease re-emergence for $90 \%$ of the surgically treated patients[9]. In contrast with most cancers, which have seen remarkable improvements in survival rates in the last 40 years, PDAC therapy has produced only marginal improvements in survival within the same timeframe[6].

Curing PDAC has remained elusive partly due to the concomitant presence of multiple genetic alterations in PDAC patients[10, 11]. The most frequent of them is the activation of the Kras protooncogene, found in over 95\% of human PDACs; no other human cancer is more often affected by activating mutations in Kras or in any other Ras gene than PDAC[5]. Oncogenic Kras signalling is likely the main driving force behind PDAC, having been identified in very early, low-grade neoplastic precursor lesions, such as pancreatic intraepithelial neoplasia (PanIN)[12] (Figure 1A). In addition, activation of the Kras oncogene is accompanied by inactivation of the p16 tumour suppressor gene in over $90 \%$ of human PDACs[7]. More than 75\% of PDACs display mutations in at least three of the 
following genes: Kras, p16, p53, and SMAD4/DPC4, with mutations in the p53 tumour suppressor gene being found with a frequency in the $50-75 \%$ range and mutations in the SMAD4 gene, a mediator of TGF- $\beta$ signal transduction, in approximately $55 \%$ of PDACs[7]. Another common biomarker of PDAC is overexpression of EGFR, a transmembrane protein involved in activating the KRAS protein, with a prevalence of $40-70 \%[10]$.

The pathways affected by mutations in PDAC were shown to cluster in KRAS signalling, TGF- $\beta$ signalling, JNK signalling, integrin signalling, Wnt/Notch, Hedgehog signalling, control of G1/S phase transition, apoptosis, DNA damage control, small GTPase signalling, invasion and homophilic cell adhesion[13], while the recurrent coding driver mutations aggregate into 10 molecular mechanisms (Figure 1B)[14].

\section{Cancer stem cells and resistance to therapy}

A factor that has emerged as showing great potential clinical significance in PDAC is tumour heterogeneity. PDAC cells vary in their tumourigenic capabilities, and PDAC propagation and growth has been shown to be highly reliant upon the presence of a restricted subset of PDAC cells, known as pancreatic cancer stem cells (CSCs) $[15,16]$. The term "cancer stem cells" is used in reference to two characteristics of these cells, shared with normal stem cells: self-renewal, and the ability to produce differentiated progeny[15, 16](see also Text box 1 ). Annihilating CSCs is a necessary condition that any curative therapy for PDAC needs to satisfy - one single surviving CSC is theoretically able to regenerate the whole tumour[8].

CSCs have been conclusively shown to be involved in PDAC resistance to chemotherapy, displaying increased prevalence within the tumour after treatment with gemcitabine[17]. While the mechanism of pancreatic CSCs resistance to cytotoxic chemotherapy is multifaceted, the relative quiescence of CSCs is an important aspect of it[18]. This quiescence is thought to be governed by epigenetic and 
metabolic cues from the tumour microenvironment, and p53 has been implicated in the process[18]. The epigenetic alterations of pancreatic CSCs include higher overall DNA methylation levels than cancer non-stem cells, and inhibition of an enzyme linked to this feature, DNA methyltransferase 1, was shown to both restrict quiescence and induce differentiation[18]. In the case of PDAC, the increased methylation is observed especially in the non-CpG islands and intergenic regions[19]. JNK is upregulated in pancreatic CSCs and contributes to their maintenance while it may also contribute to the chemoresistance of CSCs through prevention of 5-fluorouracil and gemcitabine-induced increase in intracellular ROS. Hence, JNK inhibition combined with 5-fluorouracil and/or gemcitabine-based regimens may be a rational therapeutic approach to effectively eliminate $\operatorname{cscs}[20]$. The mTOR pathway is also involved in PDAC chemoresistance by reprogramming energy metabolism through amino acid sensing. The L-type amino acid transporter 2 (LAT2) functions as an oncogenic protein and regulates glutamine-dependent mTOR activation to promote glycolysis and decrease gemcitabine sensitivity in pancreatic cancer. In turn, gemcitabine combined with an mTOR inhibitor (RAD001) can increase the chemosensitivity of pancreatic cancer cells[21]. Furthermore, microRNAs (miRNA) regulate various molecular mechanisms of PDAC, for instance miR-1246 expression has been associated with chemoresistance and CSC-like properties via CCNG2, and could predict worse prognosis in patients[22]. Remarkably, in the case of radiation therapy, dying pancreatic CSCs were shown to secrete the high-mobility group box 1 (HMGB1) protein, which binds the Toll-like receptor 2 (TLR-2) of living PDAC cells, triggering invasive and metastatic behaviour through the activation of the PI3K-Akt pathway[23].

A few recent clinical trials, detailed in the corresponding section, have tried to target CSCs directly. Two distinct signalling pathways involved in the maintenance of CSCs were targeted so far, Hedgehog and JAK-STAT, but the results were unsatisfactory. If the hypothesis that CSC can emerge from cancer non-stem cells is correct in the case of PDAC, a significant amount of work should be devoted to attempting not only to eliminate pre-existing CSCs - as the two approaches mentioned above did but also to eliminate CSCs arising from cancer non-stem cells[6]. 


\section{Molecular targets of proposed drugs}

120 A range of molecular therapeutic approaches have been attempted in the context of PDAC (Figure 2).

121 These therapeutic approaches are grouped here by their mechanisms of action. We discuss targeted

122 therapy below while currently used drugs mostly falling under cytotoxic therapy is discussed in Text 123 box 2.

\section{Targeted therapy}

The classification scheme we use for targeted therapy involves the main cellular processes that characterise cancer cells and the tumourigenic process in general (Figure 3).

Targeting the sustained proliferative signalling

Dysregulation of growth factor receptors and their associated pathways is a feature shared by many cancers.

Epidermal growth factor (EGF) is one of the most important regulators of cell survival. It can activate a wide range of signalling pathways, most notably PI3K/Akt, Ras/Raf/MEK/ERK and Jak-STAT[24]. EGFR plays an essential role in the tumourigenesis of PDAC: EGFR activity is required for the KRAS activation of ERK, which induces tumour formation[25]. It is one of the earliest pathways deregulated in PDAC and hence of interest as a therapeutic target. A Phase III study found that addition of erlotinib leads to more significant survival improvements in PDAC patients with a wild-type KRAS gene[26], which is still present only in a small fraction of PDAC tumours. 
141 The Ras/Raf/MEK/ERK pathway mediates the cellular response to the action of several growth factors. In PDAC, most attempts to target this essential pathway have acted downstream of the typically mutated KRAS, traditionally considered an inaccessible drug target[11]. Upstream of KRAS, using farnesyltransferase inhibitors has been unsuccessful, due to a compensatory mechanism involving the geranylgeranyltransferase enzyme[5]. On the other hand, the autophagy inhibitor chloroquine and genetic or pharmacologic inhibition of specific autophagy regulators synergistically enhanced the ability of ERK inhibitors to mediate antitumour activity in KRAS-driven PDAC[27]. An exciting development of the past few years is the emergence of inhibitors that target KRAS directly, defying the long-held assumption that KRAS was undruggable. While these inhibitors (AMG 510 and MRTX8) target only the G12C mutant variant of KRAS[28, 29] and only 1-4\% of PDACs display this mutation[30], the progress is encouraging.

The phosphatidylinositol-3-kinase (PI3K)/Akt and the mammalian target of rapamycin (mTOR) signalling pathways exert essential control over multiple process related to cell growth and survival in both health and disease[31]. The pathway also plays a variety of roles in cell adhesion, migration and invasion[32]. However, inhibitors of this pathway have rarely figured in clinical trials for PDAC.

The Wnt pathway is involved in tissue development in embryos, and tissue maintenance in adults. Aberrant activation of this pathway has been associated with cancer, especially those affecting the digestive tract. Furthermore, activation of this pathway is strongly associated with CSCs[33]. Wnt inhibitor vantictumab, a monoclonal antibody targeting the Frizzled receptor, showed encouraging tumour activity in combination with gemcitabine, although safety concerns have been raised[34]. 
The stem cell factor (SCF) supports the proliferation and differentiation of cells expressing its ligand, the c-Kit tyrosine kinase. The presence of c-Kit has been documented in several PDAC cell lines. Masitinib, which inhibits both the SCF and the PDGF pathway, delivered encouraging results in combination with gemcitabine[35], showing efficiency in patients with overexpression of the ACOX1 marker.

Hedgehog pathway inhibitors

The Hedgehog pathway can regulate developmental processes and cell differentiation and has been found to be hyperactivated in PDAC. This pathway induces the expression of cell cycle regulators such as Cyclin D1 and B1, angiogenic factors such as angiopoietins and anti-tumorigenic factors while decreasing pro-apoptotic genes. However, Phase II clinical trials did not show clinical benefits in $\operatorname{PDAC}[36,37]$.

\section{Targeting Myc}

The therapeutic targeting of Myc is an attractive avenue in the light of recent findings which showed in a mouse model that MYC acts as a reversible driver of PDAC while abrogation of MYC leads to rapid regression of the ensemble tumour through both cancer cell-intrinsic and cancer cell-extrinsic mechanisms $[38,39]$. Interestingly, activation of PP2A and inhibition of mTOR synergistically reduce MYC signalling and decrease PDAC tumour growth[40].

\section{Evading growth suppressors}

\section{Aurora kinase inhibitors}

The Aurora kinase family fulfils important roles in cell division, and these kinases have been found overexpressed in a number of tumours. In PDAC, danusertib, a pan-Aurora kinase inhibitor with activity against other kinases such as RET, showed very limited clinical responses[41]. 
Cyclin-dependent kinases (CDK) are crucial regulators of the cell cycle and of RNA transcription, universally overactivated in cancer. In PDAC, clinical trials have failed to show the desired results. However, an encouraging mouse xenograft study showed that CDK4/6 inhibitors prevent recovery of PDAC cells damaged after taxane-based chemotherapy[42].

\section{Angiogenesis inhibitors}

\section{VEGF pathway inhibitors}

The vascular endothelial growth factor (VEGF) pathway is a key regulator of angiogenesis. Inhibitors of this pathway have been the subject of numerous clinical trials in PDAC, but with few or no clinical benefits. The combination of VEGF inhibitor bevacizumab with low-dose 5-fluorouracil, leucovorin, nab-paclitaxel and oxaliplatin originally reported a remarkable overall survival of more than 17 months, with acceptable toxicity. A retrospective analysis placed the overall survival even slightly higher, in the region of 19 months[43]. Moreover, the 1-year survival rate was an impressive $82 \%$, far exceeding the 1-year survival rate of accepted therapies (35-48\%)[43].

\section{Invasion and metastasis inhibitors}

\section{TGF-8 pathway inhibitors}

The TGF- $\beta$ signalling pathway plays diverse roles in cell proliferation, cell differentiation, migration and apoptosis. It can act both as a tumour suppressor and as a tumour promoter; TGF- $\beta$ is one of the most potent inhibitors of cell proliferation in normal cells, but a marker of metastasis and decreased survival in cancer, where it is thought to assist the advancement of existing tumours. Altered TGF- $\beta$ signalling bears an important role in the carcinogenesis of PDAC[44]. Two TGF- $\beta$ signalling inhibitors, trabedersen and galunisertib, have entered clinical trials for PDAC. Trabedersen, an antisense 
nucleotide inhibitor of TGF- $\beta 2[45]$ delivered encouraging results in a Phase II monotherapy trial.

Galunisertib, an inhibitor of the ALK5 serine/threonine kinase showed similarly promising activity in combination with gemcitabine[46, 47].

\section{Inhibitors of resisting apoptosis}

217 The insulin-like growth factor (IGF) pathway induces cell proliferation, represses programmed cell 218 death, and promotes cell differentiation[48]. The latter effect is mediated by the Ras/MAPK pathway. 219 Deregulated IGF signalling is a feature shared by numerous cancers, including PDAC. Furthermore, the pathway plays a role in the maintenance of CSCs: IGF signalling promotes CSC resistance to gefitinib in EGFR-mutated non-small cell lung cancer, CSC resistance to radiotherapy in glioma, and CSC selfrenewal in pancreatic and hepatic cancer[48]. The most notable result in PDAC has been obtained for the IGF-1R antagonist, dalotuzumab, which improved overall survival but not progression-free survival together with gemcitabine and erlotinib[49].

The NF-KB pathway induces the expression of inhibitors of apoptosis (IAPs), as well of some member of the Bcl-2 family, proteins with anti-apoptotic role. It also downregulates p53 expression, induces expression of matrix metalloproteases - proteins with a role in invasion, and is overall thought to play a significant role in tumorigenesis. NF-KB inhibitors curcumin and theracurmin, a more bioavailable formulation of curcumin, gave promising results in a clinical trial[50]. 
Metabolic targeting has provided intriguing discoveries in recent years. Cysteine depletion has been shown to induce pancreatic tumour ferroptosis, a form of cell death that results from the catastrophic accumulation of lipid reactive oxygen species (ROS). Administration of cyst(e)inase, a drug that depletes cysteine and cystine, has demonstrated a translatable means to induce ferroptosis in PDAC[51]. Stromal-cancer metabolic niches can form through differential transporter expression, creating unique therapeutic opportunities to target metabolic demands of cancer. For instance, targeting PDAC-specific alanine uptake by SLC38A2 impairs tumour growth[52]. Interestingly, tumourassociated macrophages (TAMs) release a spectrum of pyrimidine species including deoxycytidine, which inhibits gemcitabine through molecular competition at the level of drug uptake and metabolism. Depletion of TAMs in murine models of PDAC sensitizes these tumours to gemcitabine[53].

\section{HIF-1 pathway inhibitors}

Hypoxia-inducible factor 1 (HIF-1) plays a critical role in the pathogenesis and progression of PDAC, with a key role in triggering metastasis[54]. Inhibiting HIF-1 might serve to improve PDAC's unfavourable hypoxic and immunosuppressive microenvironment[54], and has been shown to reduce the prevalence of cells expressing the CSC marker CD133 in PDAC[55]. In immunocompetent mice, combining the HIF-1 inhibitor PX-478 with gemcitabine showed significant antitumour activity and increased the proportion of tumour-infiltrating T-lymphocytes[54]. PX-478 also showed activity in PDAC in combination with arsenic trioxide, evidenced through the induction of apoptosis through the mediation of ROS in a mouse xenograft model.

The redox master regulator NRF2 can stimulate PDAC tumourigenesis, by acting as a mediator of oncogenic KRAS, and is thought to be important to maintenance of PDAC as well, through its effects upon EGFR signalling[56]. In a mouse xenograft model, the NRF2 inhibitor PIK-75 augmented the antitumour effects of gemcitabine[57]. Of note, the function of antioxidants and the NRF pathway is 
likely to have contrasting effects on PDAC depending on its developmental stage and hence warrants careful consideration for targeting the redox regulatory system in PDAC: lower levels of ROS promote tumour initiation in the premalignant condition and higher levels of ROS promote metastatic progression[58].

\section{PKCD-PKD1 pathway inhibitors}

Mutant KRAS-Induced mitochondrial ROS in acinar cells upregulates EGFR signalling through NFKB1/NFkB2 to drive formation of pancreatic precancerous lesions[59]. To avoid cytotoxic signalling and to facilitate tumourigenesis, cancer cells have mechanisms that upregulate antioxidant genes by sensing increased ROS. Protein kinase D1 (PKD1), the serine/threonine kinase, is one of these ROS sensors[60]. PKC $\delta$ is an activating kinase of PKD1, which in turn regulates mitochondrial depolarization and ROS production[61]. Hence, targeting this signalling pathway could be used to elevate ROS to toxic levels in cancer cells.

$\alpha$-glucosidase inhibitors

Terphenyllin is a naturally abundant $p$-terphenyl metabolite isolated from the coral derived fungus Aspergillus candidus that has $\alpha$-glucosidase inhibitory activity and was shown to inhibit PDAC growth and metastasis in a mouse model by inducing apoptosis through Bax, Bad and Puma[62].

\section{Anti-inflammatory drugs}

\section{JAK-STAT pathway inhibitors}

The JAK/STAT3 pathway plays an important role in cell proliferation, angiogenesis and apoptosis, among other functions[63]. The JAK (Janus kinase) constitute a family of non-transmembrane tyrosine kinases[63]. The STAT protein family includes 7 different members; of these, STAT3 and STAT5 are thought to have the highest oncogenic potential. Moreover, STAT3 is important to the emergence of 
CSCs from non-stem cancer cells, through the IL-6-JAK1-STAT3-Oct4 pathway[64], and also mediates the maintenance of p21 activated kinase 4 (PAK4)-induced CSCs in PDAC[65].

Deregulation of the Src tyrosine kinase in cancer is thought to enhance tumour growth and invasive behaviour. However, a Src inhibitor, dasatinib, failed in Phase II trials for PDAC, both as monotherapy and in combination with gemcitabine[66, 67].

Cyclooxygenase-2 (COX-2) has been found overexpressed in $75 \%$ of resected pancreatic tumours, and it correlates with aggressive and invasive tumour behaviour. It is commonly thought to be important to carcinogenesis by indirectly inhibiting apoptosis and stimulating angiogenesis. Nevertheless, PDAC Phase II clinical trials of a COX-2 inhibitor, celecoxib, have not shown satisfactory results.

In 2016 Bruton tyrosine kinase (BTK) was found to restore the T-cells-driven antitumour immune response in the context of PDAC[68], and it therefore became regarded as a promising target in this disease. However, BTK inhibitors ibrutinib[69] and acalabrutinib[70] failed in clinical trials.

\section{Drugs causing cell death due to genome instability and DNA damage}

Poly (ADP-ribose) polymerase (PARP) inhibitors target cancer cells with a homologous recombination repair (HRR) deficiency, such as BRCA gene mutation, due to synthetic lethality. PARP inhibitors (olaparib, rucaparib and niraparib) can trap the PARP-1 protein at a single-stranded break/DNA lesion and disrupt its catalytic cycle, ultimately leading to replication fork progression and consequent double-strand breaks[71]. Tumour cells with BRCA mutations will die due to HRR loss. The POLO 
304 (Pancreatic Cancer Olaparib Ongoing) trial has demonstrated a longer median progression-free survival for olaparib group, and rucaparib has also performed encouragingly in combination with gemcitabine[72].

\section{Therapeutics promoting immune destruction}

Immunotherapy is a promising strategy for various cancers while PDAC are regarded as immunequiescent and non-responsive to single-checkpoint blockade therapies. Among other mechanisms, this non-responsiveness involves CSC characteristics of PDAC cells, since the CSC marker doublecortin calmodulin-like kinase 1 (DCLK1) increases invasion, drug resistance, the activation of PI3K/AKT/MTOR-pathway signalling and KRAS activity[73]. DCLK1 also regulates the level of PD-L1 expression and tumour immunity by affecting the corresponding expression level of yes-associated protein in the Hippo pathway[74].

Autophagy plays an important function in immune evasion. PDAC cells have reduced expression of MHC-I at the cell surface due to lysosomal degradation by an autophagy-dependent mechanism. Inhibition of autophagy, either genetically or pharmacologically with chloroquine, synergizes with dual immune checkpoint blockade therapy (anti-PD1 and anti-CTLA4 antibodies), and leads to an enhanced anti-tumour immune response[75]. The addition of hydroxychloroquine to preoperative gemcitabine and nab-paclitaxel chemotherapy in patients with resectable PDAC resulted in greater pathologic tumour response, improved serum biomarker response, and evidence of autophagy inhibition and immune activity[76]. Besides the now FDA-approved pembrolizumab, an inhibitor of PD-L1 and an inhibitor of another immune checkpoint protein, CTLA-4, showed promising results in a Phase II trial when administered in combination with gemcitabine and nab-paclitaxel[77]. macrophages an important therapeutic target. The combination of an agonist CD40 antibody with 
gemcitabine led to tumour regressions in some patients. Interestingly, in mice, CD40-activated macrophages rapidly infiltrated tumours, became tumouricidal, and facilitated the depletion of tumour stroma[78]. Therefore, a CD40-dependent mechanism for targeting tumour stroma in the treatment of PDAC is a promising route[79]. Furthermore, metabolic rewiring of macrophages by CpG oligodeoxynucleotide, a Toll-like receptor 9 agonist, can potentiate clearance of cancer cells and overcomes tumour-expressed CD47-mediated signal[80].

During development of pancreatic cancer, alternatively activated macrophages contribute to fibrogenesis, PanINs, and generation of an immunosuppressive environment. The immunomodulatory agent pomalidomide depletes pancreatic lesion areas of alternatively activated macrophage populations indicating that pomalidomide could be used to decrease fibrogenesis in pancreatic cancer[81].

Interleukin-13 alters macrophage populations and mediates pancreatic tumourigenesis[82] while agonism of the integrin molecule CD11b/CD18 reprograms innate immunity to sensitize pancreatic cancer to immunotherapies[83]. Macrophages can also support CSCs, since macrophage-expressed CD51 promotes CSC properties via the TGF- $\beta 1 / \mathrm{Smad} 2 / 3$ axis indicating that CD51-targeted therapy could be a therapeutic strategy for eliminating $\operatorname{CSCs}[84]$.

The novelty of the immunotherapy approach suggests that clinical trials of immunotherapeutic drugs will continue, as only a small portion of the immunotherapeutic landscape has been explored in the context of this disease.

\section{Targeting epigenetic mechanisms}

Gene expression driven by epigenetic changes plays a crucial role in both the onset and the progression of cancer. The developmental plasticity that characterises CSCS and their ability to give rise to new tumours in their entire cellular heterogeneity, is an important mechanism for metastatic 
dissemination and therapeutic resistance. One application of using therapeutics that target epigenetic mechanisms could be to abolish the stem cell-like characteristics that control cellular plasticity, thereby making the CSCs more prone to other combined therapies (Figure 4A). Targeting the stem cell factors and their complexes with epigenetic machineries would not only antagonize the growth of the primary tumour but also block metastatic derivatives. This strategy would aim to reverse the developmental plasticity of CSCS and trap it to a more restricted cell fate that would re-sensitise these cancer cells to combined chemotherapeutic treatments. This would target the epigenetic instability and the emergence of CSCs during tumour evolution and formation of therapeutic resistance. For instance, histone lysine demethylase $3 \mathrm{~A}(\mathrm{KDM} 3 \mathrm{~A})$ is upregulated in PDAC and promotes colony formation, invasion, migration, and spheroid formation as well as orthotopic tumours in mice. Interestingly, hypoxia increases KDM3A which in turn upregulates CSC marker DCLK1, indicating that this pathway could serve as a potential target for treatment of pancreatic cancer in future studies[85, 86]. Currently, novel epigenetic targets are under investigation but reliable data on specific targeting of CSCs is lacking.

Histone deacetylase (HDAC) inhibitors

Since their inhibition can reverse epigenetic states linked with cancer, HDACs are attractive targets in cancer. However, in the context of PDAC, two HDAC inhibitors, mocetinostat and panobinostat, failed in Phase $I[87,88]$.

Bromodomain inhibitors been described as promising anti-cancer agents[89]. In PDAC, bromodomain inhibitor I-BET 672 showed antitumour activity[90] and synergy with gemcitabine[91]. 
Desmoplasia, understood as the abundance of fibrotic stroma, is a feature typical of PDAC and of important clinical interest. The fibrotic stroma is derived mainly from pancreatic stellate cells[92]. Desmoplasia is commonly observed around pre-cancerous pancreatic lesions and it can account for as much as $80 \%$ of the tumour mass in PDAC.

The stroma is thought to be a barrier which hinders the delivery of therapeutic agents in human PDAC (Figure 4B). This is believed to occur, ultimately, through the action of collagens, laminin and fibronectin - secreted by pancreatic stellate cells, they contribute to the enhanced stiffness of the tumour microenvironment, as well as to its elevated hyaluronic acid content and hydrostatic pressures. Consequently, therapeutic approaches that involve the concurrent targeting of the stroma (for example, through antifibrotic agents), and the direct targeting of PDAC cells, either through chemotherapy or targeted therapy, is a potentially viable route towards improved treatment.

An aberrant wound-healing process accompanies PDAC cell-induced transdifferentiation of tissueresident pancreatic stellate cells (PSC) into activated cancer-associated fibroblasts (CAFs). CAFs are heterogenous and can have both tumour-supportive and tumour-suppressive or homeostatic function[93]. Among CAFs, an MHC class II-expressing population presents antigens to $\mathrm{CD}^{+} \mathrm{T}$ cells and can potentially modulate the immune response in pancreatic tumours[94]. In addition, CAFs activate the wound-healing autotaxin-lysophosphatidic acid axis that promotes PDAC cell proliferation, migration, and AKT activation, while genetic or pharmacologic autotaxin inhibition suppresses PDAC growth in vivo[95].

Targeting mucins has recently been suggested as a therapeutic avenue because the glycan CA19-9 promotes pancreatitis with hyperactivation of EGFR signalling and pancreatic cancer in mice[96]. Interestingly, Vitamin D analogues could also be very beneficial in targeting stroma since Vitamin D receptor-mediated stromal reprogramming suppresses pancreatitis and enhances pancreatic cancer therapy[97]. Pegvorhyaluronidase alfa (PEGPH20), an enzyme which degrades hyaluronic acid, did not improve survival[98]. 
In a preclinical setting, stromal depletion - after the administration of FAK inhibitors in mice - resulted in treatment resistance through the activation of STAT3 signalling[99]. However, concurrent administration of Src and EGFR inhibitors led to both inhibition of STAT3 signalling and stromal remodelling[100]. In addition, halofuginone assists enhanced drug transport and the recruitment of favourable immune cells through its effects upon the stroma[101].

The delivery of therapeutic compounds to tumours could be enhanced by ultrasound-triggered targeting[102]. Ultrasonically driven bubbles (cavitation) can overcome intratumoural pressure and enhance the delivery, extravasation and penetration of macromolecular drugs into tumours. Such bubbles driven in a sustained inertial cavitation regime are able to actively transport and enhance the distribution of therapeutic macromolecules to more than 100 micrometers from the nearest blood vessel[103] which would be beneficial for PDAC due to its hypovascularisation.

Collectively, the combined targeting of stroma together with compounds that are directed against CSCs and cancer non-stem cells, could be a more efficient strategy in PDAC than individual treatment options.

\section{Concluding Remarks and Future Perspectives}

The analysis of clinical trial data shows a clear shift from chemotherapy towards targeted therapy although classical chemotherapy might still play a useful role in combination with targeted agents. The exploration of inhibitors of proliferative signalling is far from complete and some of the trials have shown encouraging results. Most notably, the direct inhibition of KRAS is a major and unprecedented step forward - even though it has only been achieved yet for the G12C-mutated variant of KRAS, known to be rare in PDAC. Furthermore, the TGF- $\beta$ pathway is quite likely to receive a significant amount of clinical attention in the following years, while c-Kit, HIF-1, NF-KB and NRF2 pathways could also be attractive candidates (see Outstanding questions). 
425 An encouraging note also comes from immunotherapy, which has defeated some of the initial scepticism given by the context of the immunosuppressive tumour microenvironment characteristic of PDAC. Targeting the stroma together with other strategies will probably be the subject of more clinical trials.

Similarly, epigenetic approaches are likely to become more prominent. The 2020 FDA approval of zeste homolog $2(E Z H 2)$ inhibitor tazememostat is the first approval of an epigenetic drug for a solid tumour[104], after more than a decade in which all epigenetic cancer drugs were used only to treat haematological malignancies.

Finally, due to the abundant evidence of the formation of CSCs or tumour initiating cells in a range of cancer including PDAC, the developmental plasticity and stem cell-like characteristics of some cancer cells can be regarded as one of the hallmarks of cancer. It is clear that annihilating CSCs will remain a crucial prerequisite of attaining success in PDAC therapeutics. The failure of therapies that aimed to eliminate them directly - through targeting JAK-STAT and Hedgehog pathways, suggests that new approaches are needed. As the concept of CSCs gets more and more refined, we expect that the therapies targeting them will also become more and more effective, until the elimination of these cells eventually becomes a realizable goal.

\section{Acknowledgements}

This work in S.P.'s laboratory was supported by a Cancer Research UK Career Development Fellowship,

444 Grant ID C59392/A25064, an ESPRC studentship (A-F.S), and Daphne Jackson Fellow funded by The 445 Kennedy Trust for Rheumatology Research (CH.C). 
448 The authors declare no conflicts of interest.

449

450

451

452

453

454

455

456

457

458

459

460

461

462

463

464 


\section{References}

466

467

468

469

470

471

472

473

474

475

476

477

478

479

480

481

482

483

484

485

486

487

488

489

1. Hill, A. and Chung, V. (2020) Pancreatic cancer. In Oncology in the Precision Medicine Era, pp. 97109, Springer.

2. Zhang, Y. et al. (2018) Novel agents for pancreatic ductal adenocarcinoma: emerging therapeutics and future directions. Journal of hematology \& oncology 11 (1), 14.

3. Palchetti, S. et al. (2019) Protein corona fingerprints of liposomes: New opportunities for targeted drug delivery and early detection in pancreatic cancer. Pharmaceutics $11(1), 31$.

4. Ying, H. et al. (2016) Genetics and biology of pancreatic ductal adenocarcinoma. Genes Dev 30 (4), $355-85$.

5. Choi, M. et al., Challenges in Ras therapeutics in pancreatic cancer, Seminars in cancer biology, Elsevier, 2019, pp. 101-108.

6. Smigiel, J.M. et al. (2018) Targeting pancreatic cancer cell plasticity: the latest in therapeutics. Cancers 10 (1), 14.

7. Li, J. et al. (2010) Pancreatic cancer: pathobiology, treatment options, and drug delivery. AAPS J 12 (2), 223-32.

8. Hermann, P.C. and Sainz Jr, B., Pancreatic cancer stem cells: A state or an entity?, Seminars in cancer biology, Elsevier, 2018, pp. 223-231.

9. Sinn, M. et al. (2017) CONKO-005: Adjuvant Chemotherapy With Gemcitabine Plus Erlotinib Versus Gemcitabine Alone in Patients After Ro Resection of Pancreatic Cancer: A Multicenter Randomized Phase III Trial. J Clin Oncol 35 (29), 3330-3337.

10. Karandish, F. and Mallik, S. (2016) Biomarkers and Targeted Therapy in Pancreatic Cancer. Biomark Cancer 8 (Suppl 1), 27-35.

11. Michael, J.V. and Goldfinger, L.E., Concepts and advances in cancer therapeutic vulnerabilities in RAS membrane targeting, Seminars in cancer biology, Elsevier, 2019, pp. 121-130.

12. Eser, S. et al. (2014) Oncogenic KRAS signalling in pancreatic cancer. Br J Cancer 111 (5), 817-22. 
13. Jones, S. et al. (2008) Core signaling pathways in human pancreatic cancers revealed by global genomic analyses. Science 321 (5897), 1801-6.

14. Bailey, P. et al. (2016) Genomic analyses identify molecular subtypes of pancreatic cancer. Nature 531 (7592), 47-52.

15. Li, C. et al. (2007) Identification of pancreatic cancer stem cells. Cancer Res 67 (3), 1030-7.

16. Hermann, P.C. et al. (2007) Distinct populations of cancer stem cells determine tumor growth and metastatic activity in human pancreatic cancer. Cell Stem Cell 1 (3), 313-23.

17. Mueller, M.T. et al. (2009) Combined targeted treatment to eliminate tumorigenic cancer stem cells in human pancreatic cancer. Gastroenterology 137 (3), 1102-13.

18. Lanfranca, M.P. et al., Metabolism and epigenetics of pancreatic cancer stem cells, Seminars in cancer biology, Elsevier, 2019, pp. 19-26.

19. Zagorac, S. et al. (2016) DNMT1 Inhibition Reprograms Pancreatic Cancer Stem Cells via Upregulation of the miR-17-92 Cluster. Cancer Res 76 (15), 4546-58.

20. Suzuki, S. et al. (2015) JNK suppression of chemotherapeutic agents-induced ROS confers chemoresistance on pancreatic cancer stem cells. Oncotarget 6 (1), 458.

21. Feng, M. et al. (2018) LAT2 regulates glutamine-dependent mTOR activation to promote glycolysis and chemoresistance in pancreatic cancer. Journal of Experimental \& Clinical Cancer Research 37 (1), 274.

22. Hasegawa, S. et al. (2014) MicroRNA-1246 expression associated with CCNG2-mediated chemoresistance and stemness in pancreatic cancer. British journal of cancer 111 (8), 1572-1580. 23. Chen, X. et al. (2018) Radiotherapy-induced cell death activates paracrine HMGB1-TLR2 signaling and accelerates pancreatic carcinoma metastasis. Journal of Experimental \& Clinical Cancer Research $37(1), 77$.

24. Fitzgerald, T.L. et al. (2015) Roles of EGFR and KRAS and their downstream signaling pathways in pancreatic cancer and pancreatic cancer stem cells. Advances in biological regulation 59, 65-81. 

Cancer cell 22 (3), 304-317. 26. Boeck, S. et al. (2013) EGFR pathway biomarkers in erlotinib-treated patients with advanced pancreatic cancer: translational results from the randomised, crossover phase 3 trial AIO-PK0104. British journal of cancer 108 (2), 469-476. 27. Bryant, K.L. et al. (2019) Combination of ERK and autophagy inhibition as a treatment approach for pancreatic cancer. Nature medicine 25 (4), 628-640. 28. Canon, J. et al. (2019) The clinical KRAS (G12C) inhibitor AMG 510 drives anti-tumour immunity. Nature 575 (7781), 217-223. 29. Seton-Rogers, S. (2020) KRAS-G12C in the crosshairs. Nature Reviews Cancer 20 (1), 3-3. 30. Janes, M.R. et al. (2018) Targeting KRAS mutant cancers with a covalent G12C-specific inhibitor. Cell $172(3)$, 578-589. e17. 31. Porta, C. et al. (2014) Targeting PI3K/Akt/mTOR signaling in cancer. Frontiers in oncology 4, 64. 32. Karar, J. and Maity, A. (2011) PI3K/AKT/mTOR pathway in angiogenesis. Frontiers in molecular neuroscience 4, 51 . 33. Zhan, T. et al. (2017) Wnt signaling in cancer. Oncogene 36 (11), 1461-1473. 34. Davis, S.L. et al. (2019) A phase $1 \mathrm{~b}$ dose escalation study of Wnt pathway inhibitor vantictumab in combination with nab-paclitaxel and gemcitabine in patients with previously untreated metastatic pancreatic cancer. Investigational new drugs, 1-10. 35. Deplanque, G. et al. (2015) A randomized, placebo-controlled phase III trial of masitinib plus gemcitabine in the treatment of advanced pancreatic cancer. Annals of Oncology 26 (6), 1194-1200. 36. Catenacci, D.V. et al. (2015) Randomized phase Ib/ll study of gemcitabine plus placebo or 537 vismodegib, a hedgehog pathway inhibitor, in patients with metastatic pancreatic cancer. Journal of 538 Clinical Oncology 33 (36), 4284. 
539

540

541

542

543

544

545

546

547

548

549

550

551

552

553

554

555

556

557

558

559

560

561

562

563

564

37. De Jesus-Acosta, A. et al. (2020) Phase 2 study of vismodegib, a hedgehog inhibitor, combined with gemcitabine and nab-paclitaxel in patients with untreated metastatic pancreatic adenocarcinoma. British journal of cancer 122 (4), 498-505.

38. English, I.A. and Sears, R.C. (2020) Deconstructing Pancreatic Adenocarcinoma by Targeting the Conductor, MYC. Cancer discovery 10 (4), 495-497.

39. Sodir, N.M. et al. (2020) Myc instructs and maintains pancreatic adenocarcinoma phenotype. Cancer Discovery 10 (4), 588-607.

40. Allen-Petersen, B.L. et al. (2019) Activation of PP2A and inhibition of mTOR synergistically reduce MYC signaling and decrease tumor growth in pancreatic ductal adenocarcinoma. Cancer research 79 (1), 209-219.

41. Laffranchi, B. et al. (2010) Phase II study of danusertib (D) in advanced/metastatic colorectal and pancreatic cancers (CRC, PC). Journal of Clinical Oncology 28 (15_suppl), e13558-e13558.

42. Salvador-Barbero, B. et al. (2020) CDK4/6 inhibitors impair recovery from cytotoxic chemotherapy in pancreatic adenocarcinoma. Cancer Cell.

43. Isacoff, W.H. et al. (2018) Low-dose continuous 5-fluorouracil combined with leucovorin, nabpaclitaxel, oxaliplatin, and bevacizumab for patients with advanced pancreatic cancer: a retrospective analysis. Targeted oncology 13 (4), 461-468.

44. Truty, M.J. and Urrutia, R. (2007) Basics of TGF- $\beta$ and pancreatic cancer. Pancreatology 7 (5-6), 423-435.

45. Jaschinski, F. et al. (2011) The antisense oligonucleotide trabedersen (AP 12009) for the targeted inhibition of TGF- $\beta 2$. Current pharmaceutical biotechnology 12 (12), 2203-2213.

46. Melisi, D. et al., A phase II, double-blind study of galunisertib+ gemcitabine (GG) vs gemcitabine+ placebo (GP) in patients (pts) with unresectable pancreatic cancer (PC), American Society of Clinical Oncology, 2016.

47. Melisi, D. et al. (2018) Galunisertib plus gemcitabine vs. gemcitabine for first-line treatment of patients with unresectable pancreatic cancer. British journal of cancer 119 (10), 1208-1214. 
565

566

567

568

569

570

571

572

573

574

575

576

577

578

579

580

581

582

583

584

585

586

587

588

589

590

48. Denduluri, S.K. et al. (2015) Insulin-like growth factor (IGF) signaling in tumorigenesis and the development of cancer drug resistance. Genes \& diseases 2 (1), 13-25.

49. Abdel-Wahab, R. et al. (2018) Randomized, phase I/II study of gemcitabine plus IGF-1R antagonist (MK-0646) versus gemcitabine plus erlotinib with and without MK-0646 for advanced pancreatic adenocarcinoma. Journal of hematology \& oncology 11 (1), 71.

50. Imaizumi, A. (2015) Highly bioavailable curcumin (Theracurmin): its development and clinical application. PharmaNutrition 3 (4), 123-130.

51. Badgley, M.A. et al. (2020) Cysteine depletion induces pancreatic tumor ferroptosis in mice. Science 368 (6486), 85-89.

52. Parker, S.J. et al. (2020) Selective alanine transporter utilization creates a targetable metabolic niche in pancreatic cancer. Cancer Discovery.

53. Halbrook, C.J. et al. (2019) Macrophage-released pyrimidines inhibit gemcitabine therapy in pancreatic cancer. Cell metabolism 29 (6), 1390-1399. e6.

54. Hao, J. (2015) HIF-1 is a critical target of pancreatic cancer. Oncoimmunology 4 (9), e1026535.

55. McGinn, O. et al. (2017) Inhibition of hypoxic response decreases stemness and reduces tumorigenic signaling due to impaired assembly of HIF1 transcription complex in pancreatic cancer. Scientific reports 7 (1), 1-11.

56. Chio, I.I.C. et al. (2016) NRF2 promotes tumor maintenance by modulating mRNA translation in pancreatic cancer. Cell 166 (4), 963-976.

57. Duong, H.-Q. et al. (2014) Inhibition of NRF2 by PIK-75 augments sensitivity of pancreatic cancer cells to gemcitabine. International journal of oncology 44 (3), 959-969.

58. Cheung, E.C. et al. (2020) Dynamic ROS control by TIGAR regulates the initiation and progression of pancreatic cancer. Cancer cell 37 (2), 168-182. e4.

59. Liou, G.-Y. et al. (2016) Mutant KRas-induced mitochondrial oxidative stress in acinar cells upregulates EGFR signaling to drive formation of pancreatic precancerous lesions. Cell reports $14(10)$, 2325-2336. 

kinase D1 and its importance in pancreatic cancer. Frontiers in oncology 7, 41. 61. Zhang, T. et al. (2015) PKD1 protein is involved in reactive oxygen species-mediated mitochondrial depolarization in cooperation with protein kinase C $\delta$ (PKC $\delta$ ). Journal of Biological Chemistry 290 (16), $10472-10485$.

62. Zhang, J. et al. (2020) Terphenyllin Suppresses Orthotopic Pancreatic Tumor Growth and Prevents Metastasis in Mice. Frontiers in Pharmacology 11, 457.

63. Hu, W., Progress of JAK/STAT 3 and its inhibitors in the treatment of cancer, AIP Conference Proceedings, AIP Publishing LLC, 2020, p. 020045. 64. Kim, S.-Y. et al. (2013) Role of the IL-6-JAK1-STAT3-Oct-4 pathway in the conversion of non-stem cancer cells into cancer stem-like cells. Cellular signalling 25 (4), 961-969.

65. Tyagi, N. et al. (2016) p-21 activated kinase 4 (PAK4) maintains stem cell-like phenotypes in pancreatic cancer cells through activation of STAT3 signaling. Cancer letters 370 (2), 260-267. 66. Evans, T. et al. (2016) Phase 2 placebo-controlled, double-blind trial of dasatinib added to gemcitabine for patients with locally-advanced pancreatic cancer. Annals of Oncology 28 (2), 354-361. 67. Chee, C.E. et al. (2013) Phase II study of dasatinib (BMS-354825) in patients with metastatic adenocarcinoma of the pancreas. The oncologist 18 (10), 1091-1092. 68. Gunderson, A.J. et al. (2016) Bruton tyrosine kinase-dependent immune cell cross-talk drives pancreas cancer. Cancer discovery 6 (3), 270-285. 69. Tempero, M. et al. (2019) O-002 Ibrutinib in combination with nab-paclitaxel and gemcitabine as

611 first-line treatment for patients with metastatic pancreatic adenocarcinoma: results from the phase 3

612 RESOLVE study. Annals of Oncology 30 (Supplement_4), mdz154. 001.

613 70. Overman, M. et al. (2020) Randomized phase II study of the Bruton tyrosine kinase inhibitor 614 acalabrutinib, alone or with pembrolizumab in patients with advanced pancreatic cancer. Journal for ImmunoTherapy of Cancer 8 (1). 

applications. Molecular cancer 19 (1), 1-15.

72. Domchek, S.M. et al., RUCAPANC: An open-label, phase 2 trial of the PARP inhibitor rucaparib in patients (pts) with pancreatic cancer (PC) and a known deleterious germline or somatic BRCA mutation, American Society of Clinical Oncology, 2016.

73. Qu, D. et al. (2019) Overexpression of DCLK1-AL increases tumor cell invasion, drug resistance, and KRAS activation and can be targeted to inhibit tumorigenesis in pancreatic cancer. Journal of oncology 2019. 74. Yan, R. et al. (2020) Inhibition of DCLK1 down-regulates PD-L1 expression through Hippo pathway in human pancreatic cancer. Life Sciences 241, 117150. 75. Yamamoto, K. et al. (2020) Autophagy promotes immune evasion of pancreatic cancer by degrading MHC-I. Nature, 1-6. 76. Zeh, H.J. et al. (2020) A randomized phase II preoperative study of autophagy inhibition with highdose hydroxychloroquine and gemcitabine/nab-paclitaxel in pancreatic cancer patients. Clinical Cancer Research. 77. Renouf, D.J. et al., The Canadian Cancer Trials Group PA. 7 trial: Results from the safety run in of a randomized phase II study of gemcitabine (GEM) and nab-paclitaxel (Nab-P) versus GEM, nab-P, durvalumab (D), and tremelimumab (T) as first-line therapy in metastatic pancreatic ductal adenocarcinoma (mPDAC), American Society of Clinical Oncology, 2018. 78. Beatty, G.L. et al. (2011) CD40 agonists alter tumor stroma and show efficacy against pancreatic carcinoma in mice and humans. Science 331 (6024), 1612-1616. 79. Vonderheide, R.H. (2020) CD40 agonist antibodies in cancer immunotherapy. Annual Review of Medicine 71, 47-58. 80. Liu, M. et al. (2019) Metabolic rewiring of macrophages by CpG potentiates clearance of cancer cells and overcomes tumor-expressed CD47- mediated 'don't-eat-me'signal. Nature immunology 20 (3), 265-275. 

immune-responsive environment at precancerous and cancerous lesions. Cancer research 79 (7), $1535-1548$.

82. Liou, G.-Y. et al. (2017) The presence of interleukin-13 at pancreatic ADM/PanIN lesions alters macrophage populations and mediates pancreatic tumorigenesis. Cell reports 19 (7), 1322-1333. 83. Panni, R.Z. et al. (2019) Agonism of CD11b reprograms innate immunity to sensitize pancreatic cancer to immunotherapies. Science translational medicine 11 (499), eaau9240. 84. Zhang, B. et al. (2019) Macrophage-expressed CD51 promotes cancer stem cell properties via the TGF- $\beta 1 /$ smad2/3 axis in pancreatic cancer. Cancer letters 459, 204-215. Tumors, Regulates Expression of DCLK1 and Promotes Tumorigenesis in Mice. Gastroenterology 157 (6), 1646-1659. e11. 86. Westphalen, C.B. et al. (2017) Functional implication of Dclk1 and Dclk1-expressing cells in cancer. Small GTPases 8 (3), 164-171.

87. Chan, E. et al. (2018) Phase I/II study of mocetinostat in combination with gemcitabine for patients with advanced pancreatic cancer and other advanced solid tumors. Cancer chemotherapy and pharmacology 81 (2), 355-364.

88. Wang, H. et al. (2012) Phase II study of panobinostat and bortezomib in patients with pancreatic cancer progressing on gemcitabine-based therapy. Anticancer research 32 (3), 1027-1031. 89. Pérez-Salvia, M. and Esteller, M. (2017) Bromodomain inhibitors and cancer therapy: From structures to applications. Epigenetics 12 (5), 323-339. 90. Leal, A.S. et al. (2017) Bromodomain inhibitors, JQ1 and I-BET 762, as potential therapies for pancreatic cancer. Cancer letters 394, 76-87. 91. Xie, F. et al. (2018) The BET inhibitor I-BET762 inhibits pancreatic ductal adenocarcinoma cell proliferation and enhances the therapeutic effect of gemcitabine. Scientific reports 8 (1), 1-10. 
93. Helms, E. et al. (2020) Fibroblast heterogeneity in the pancreatic tumor microenvironment. Cancer Discovery 10 (5), 648-656.

94. Elyada, E. et al. (2019) Cross-species single-cell analysis of pancreatic ductal adenocarcinoma reveals antigen-presenting cancer-associated fibroblasts. Cancer discovery 9 (8), 1102-1123.

95. Auciello, F.R. et al. (2019) A stromal lysolipid-autotaxin signaling axis promotes pancreatic tumor progression. Cancer discovery 9 (5), 617-627.

96. Engle, D.D. et al. (2019) The glycan CA19-9 promotes pancreatitis and pancreatic cancer in mice. Science 364 (6446), 1156-1162.

97. Sherman, M.H. et al. (2014) Vitamin D receptor-mediated stromal reprogramming suppresses pancreatitis and enhances pancreatic cancer therapy. Cell 159 (1), 80-93.

98. Hakim, N. et al. (2019) Why HALO 301 Failed and Implications for Treatment of Pancreatic Cancer. 99. Jiang, H. et al. (2020) Development of resistance to FAK inhibition in pancreatic cancer is linked to stromal depletion. Gut 69 (1), 122-132.

100. Dosch, A.R. et al. (2020) Combined Src/EGFR inhibition targets STAT3 signaling and induces stromal remodeling to improve survival in pancreatic cancer. Molecular Cancer Research.

101. Huang, H. and Brekken, R.A. (2019) The next wave of stroma-targeting therapy in pancreatic cancer. Cancer research 79 (2), 328-330.

102. Lyon, P.C. et al. (2018) Safety and feasibility of ultrasound-triggered targeted drug delivery of doxorubicin from thermosensitive liposomes in liver tumours (TARDOX): a single-centre, open-label, phase 1 trial. The Lancet Oncology 19 (8), 1027-1039.

103. Bazan-Peregrino, M. et al. (2013) Cavitation-enhanced delivery of a replicating oncolytic adenovirus to tumors using focused ultrasound. Journal of Controlled Release 169 (1-2), 40-47. 104. Hoy, S.M. (2020) Tazemetostat: First Approval. Drugs, 1-9.

105. Bonnet, D. and Dick, J.E. (1997) Human acute myeloid leukemia is organized as a hierarchy that originates from a primitive hematopoietic cell. Nat Med 3 (7), 730-7. 
106. Todaro, M. et al. (2007) Colon cancer stem cells dictate tumor growth and resist cell death by production of interleukin-4. Cell Stem Cell 1 (4), 389-402.

107. Tomizawa, Y. et al. (2012) Epithelial mesenchymal transition and cancer stem cells in esophageal adenocarcinoma originating from Barrett's esophagus. Oncol Lett 3 (5), 1059-1063.

697 108. Ma, S. et al. (2007) Identification and characterization of tumorigenic liver cancer stem/progenitor cells. Gastroenterology 132 (7), 2542-56. 109. Eramo, A. et al. (2008) Identification and expansion of the tumorigenic lung cancer stem cell population. Cell Death Differ 15 (3), 504-14.

110. Alvero, A.B. et al. (2009) Molecular phenotyping of human ovarian cancer stem cells unravels the mechanisms for repair and chemoresistance. Cell Cycle 8 (1), 158-66.

111. Fioriti, D. et al. (2008) Cancer stem cells in prostate adenocarcinoma: a target for new anticancer strategies. J Cell Physiol 216 (3), 571-5.

112. Takaishi, S. et al. (2008) Gastric cancer stem cells. J Clin Oncol 26 (17), 2876-82.

113. Todaro, M. et al. (2010) Tumorigenic and metastatic activity of human thyroid cancer stem cells.

114. Stanger, B.Z. (2015) Cellular homeostasis and repair in the mammalian liver. Annual review of physiology 77, 179-200.

115. Thorel, F. et al. (2010) Conversion of adult pancreatic $\alpha$-cells to $\beta$-cells after extreme $\beta$-cell loss. Nature 464 (7292), 1149-1154. 116. Hanahan, D. and Weinberg, R.A. (2011) Hallmarks of cancer: the next generation. cell 144 (5), 646-674. 117. Al-Hajeili, M. et al. (2014) Nab-paclitaxel: potential for the treatment of advanced pancreatic cancer. OncoTargets and therapy 7, 187. 118. Pushalkar, S. et al. (2018) The pancreatic cancer microbiome promotes oncogenesis by induction of innate and adaptive immune suppression. Cancer discovery 8 (4), 403-416. 

cancer outcomes. Cell 178 (4), 795-806. e12.

720 120. Geller, L.T. et al. (2017) Potential role of intratumor bacteria in mediating tumor resistance to the

721 chemotherapeutic drug gemcitabine. Science 357 (6356), 1156-1160.

722

723

724

725

726

727

728

729

730

731

732

733

734

735

736

737

738 
740 Angiopoietin: A protein that binds chiefly to a receptor on endothelial cells and promotes the

741 maturation and stability of blood vessels.

742 Extravasation: The leakage of blood, lymph, or other fluid, such as an anticancer drug, from a blood 743 vessel or tube into the tissue around it.

744 Fibronectin: A large glycoprotein that acts as a host defence mechanism.

745 Growth factor: A substance made by the body that functions to regulate cell division and cell survival.

746 Hyaluronic acid: An acid mucopolysaccharide that acts as the binding and protective agent of the

747 ground substance of connective tissue

Laminin: A fibrous glycoprotein forming a major component of basement membranes and serving as an adhesive surface for epithelial cells.

Locally advanced cancer: Cancer that has spread from where it started to nearby tissue or lymph nodes.

Metabolic syndrome: A common combination of insulin resistance and type 2 diabetes with central obesity.

Monoclonal antibody: Antibody made from cloned organisms, i.e., all with identical DNA. The main uses of monoclonal antibodies are in specific anticancer therapy and immunosuppression.

Oncogene: A gene that is a mutated (changed) form of a gene involved in normal cell growth. Oncogenes may cause the growth of cancer cells.

Overall survival: The length of time from either the date of diagnosis or the start of treatment for a disease, such as cancer, that patients diagnosed with the disease are still alive. 
Progression-free survival: The length of time during and after the treatment of a disease, such as cancer, that a patient lives with the disease but it does not get worse.

Proto-oncogene: a normal gene that helps a cell grow.

764

765

Response rate: The percentage of patients whose cancer shrinks or disappears after treatment.

Stroma: Fibrous connective tissue or other intercellular material that forms the structural framework

Xenograft: The transplant of an organ, tissue, or cells to an individual of another species. 


\section{Text Boxes.}

783

784

785

786

787

788

789

790

791

792

793

794

795

796

797

798

799

800

801

802

803

804

\section{Text Box 1. Cancer stem cells and their developmental hierarchies}

Cancer stem cells were found in 1997, in acute myelogenous leukemia[105], and since then the existence of cancer stem cells was discovered in brain, breast[15], colon[106], esophagus[107], liver[108], lung[109], ovarian[110], prostate[111], stomach[112] and thyroid[113] cancers, among others. In the case of PDAC, the first report of cancer stem cells dates to 2007[15]. Due to the formation of cellular heterogeneity with CSCS in PDAC and in a range of other cancers the developmental plasticity and stem cell-like characteristics among cancer cells should, in our opinion, be regarded as one of the general hallmarks of cancer.

The ability of CSCs to move both up and down the differentiation hierarchy is known as cellular plasticity[8]. In normal cells, the trait was traditionally held to be exclusive to stem cells, but recent evidence has shown instances of the contrary - in fish and amphibians, terminally differentiated hepatocytes proved capable to acquire new cellular identities in response to liver damage[114], while mouse models have succeeded in inducing reprogramming of adult cells in the endocrine pancreas[115]. These studies suggest that cellular plasticity, whether partial or total, is more common than previously believed[8].

Similar considerations apply to the context of cancer. Before 2017, it was widely held that solid tumours manifested a unidirectional differential hierarchy, with CSCs at the top[8]. The implications of this model were the following: both tumour initiation and metastasis can originate only from CSCs, and tumour heterogeneity is explained exclusively through the stages of differentiation of former CSC. Moreover, CSCs were held as the only cancer cells that significantly alter their phenotype in response to stimuli, or a result of chemotherapy. However, significant challenges to this strictly hierarchical model have emerged[8]. As such, it remains to be settled whether the concept of CSC represents 
indeed a distinct entity, or rather a non-permanent state dependent upon spatial and temporal constraints.

\section{Text box 2. Drugs currently in use for PDAC and cytotoxic therapy.}

Cancer therapeutics aim to target central processes involved in tumourigenesis called cancer hallmarks. Among these frequent tumourigenic processes are sustaining proliferative signalling, evading growth suppressors, resisting cell death, enabling replicative immortality, inducing angiogenesis, activating invasion and metastasis, reprogramming of energy metabolism, creating the tumour microenvironment, inflammation, evading immune destruction and genome instability with mutations[116]. Since each of these cancer properties is connected to other hallmarks via intracellular mechanisms such as cell growth, metabolic processes and apoptosis, the impact of individual therapeutic strategies is not exclusively restricted to one cellular process but targets several hallmarks simultaneously.

Cancer therapy was dominated by surgery and radiation therapy up until into the 1960s, and thereafter 5-fluorouracil, a thymidylate synthase inhibitor, and mitomycin C, an alkylating agent. Gemcitabine, an analogue of deoxycytidine which is also a thymidylate synthase inhibitor, became the new standard of care for the disease although response rates were still modest, with a median survival rate of 5.7 months. Further therapeutic drugs include EGFR inhibitor erlotinib, FOLFIRINOX (5fluorouracil, leucovorin and nanoliposomal irinotecan) and the microtubule disassembly inhibitor nabpaclitaxel, used in combination with gemcitabine[117]. Recent years has seen FDA approval for pembrolizumab, larotrectinib, entrectinib and olaparib for rare genetic profiles.

The conventional classification of cytotoxic drugs for PDAC treatment fall under four categories: antimetabolites, topoisomerase inhibitors, DNA cross-linking agents and microtubule-targeting agents. 
831 Changes in the microbiome contribute to PDAC by inducing innate and adaptive immune suppression[118], and can impact therapeutic responsiveness. It was observed that targeting the microbiome protects against oncogenesis, reverses intratumoural immune tolerance, and enables efficacy for checkpoint-based immunotherapy. PDAC microbiome composition, which cross-talks to the gut microbiome, also influences the survival of the patients and predicts the disease mortality[119]. Microbes can influence the efficacy of cancer therapies since Gammaproteobacteria can metabolize gemcitabine into its inactive form, which is present in many PDACs[120]. Besides bacterial dysbiosis, the fungal mycobiome promotes pancreatic oncogenesis via activation of the mannose-binding lectin (MBL)[120].

840

841

842

843 
A. Pancreatic cancer development involves different stages from acinar-ductal reprogramming, PanIN 1a/b-3 lesion formation and thereafter the pancreatic ductal adenocarcinoma stage. A small subpopulation of cells acquires a stem cell-like developmentally plastic identity known as cancer stem cells that are able to efficiently metastasize to the liver and lung. The hallmark factors deregulated during PDAC development include EGFR, KRAS and telomerase activation, and p16, p53, SMAD4 and BRCA2 loss of function. The cellular and physiological processes accompanying PDAC formation are depicted in colour scheme including decrease in immune defense, increase in immune evasion, inflammation, desmoplasia, hypoxia and reactive oxygen species production.

B. The most frequently mutated genes in PDAC and their function. Schematic depiction expected biological function of the genes that are frequently mutated in pancreatic ductal adenocarcinomas. cancer hallmarks and the corresponding therapeutics that targets this process. Most therapeutics affect several cancer hallmarks simultaneously but for simplicity, only one of the cancer hallmarks is depicted to be affected by each therapeutic strategy.

Figure 3. Extracellular and intracellular signalling pathways used as targets of PDAC therapeutics. Receptors of growth factor pathways and intracellular signalling messengers are most frequently used 

signalling factors and thereby disrupt the signalling cascades.

Figure 4. Cancer stem cells and PDAC stroma.

877 A. Cancer stem cells as a cancer hallmark and therapeutic target. Cellular heterogeneity in PDAC leads to the formation of CSCs and such developmental plasticity can be considered a hallmark of cancer. Due to their ability to self-renew, metastasize and to be more resistant to conventional cancer therapeutics, targeting CSCs is of key importance for developing more efficient cancer therapeutics. The therapeutic strategies for eliminating CSCs could involve targeting stem cell factors, self-renewal and stem cell signalling pathways, cell cycle regulators and epigenetic mechanisms that regulate the stem cell-like characteristics of CSCs. These therapeutic strategies can be used in combination with therapeutics that aim to kill the bulk of the tumour represented by non-CSCs, hence eliminating the different cancer cell populations in the patient.

B. PDAC microenvironment and stromal function. PDAC cells gradually change the stroma from impairing tumourigenesis to promoting tumour formation. The microenvironment will ultimately provide a niche for CSC self-renewal and tumour growth. Tumour and stroma are in crosstalk with each other by cell-cell and paracrine interactions between cancer cells and CAFs. CAFs are heterogeneous and include myofibroblastic, inflammatory and antigen-presenting subtypes. Fibroblasts in proximity to cancer cells are induced by TGF- $\beta$ from cancer cells into myofibroblastic CAFs, producing the mechanical barrier that can be both tumour promoting and a stromal barrier against vascularisation. Strategies to target the stroma have included the use of Sonic hedgehog (SHH) inhibitors, hyaluronidase, matrix metalloproteinase (MMP) inhibitors, fibroblast activation protein (FAP) targeting agents and CXCR4 inhibitors. The binding of PD-L1 to PD-1 inhibits T cell from killing the cancer cell while immunotherapy can be achieved by blocking of PD-1 or PD-L1 that restores T-cell 
897 function. Another therapeutic target is CTLA-4. Alternatively, T cells can be engineered to target 898 cancer cells or even specific cancer subpopulations such as cancer stem cells. 
A

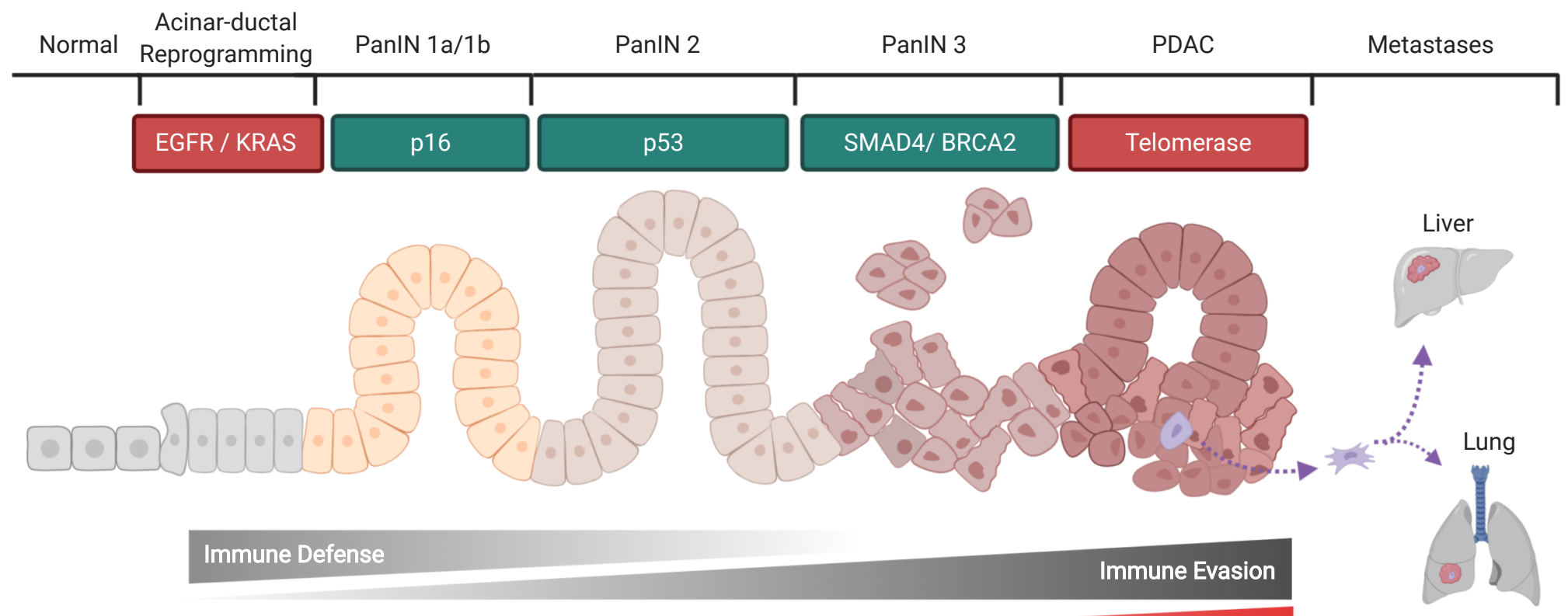

Inflammation/ Prancreatitis

Desmoplasia / Fibrosis

Hypoxia

ROS

Accumulation of mutations

B

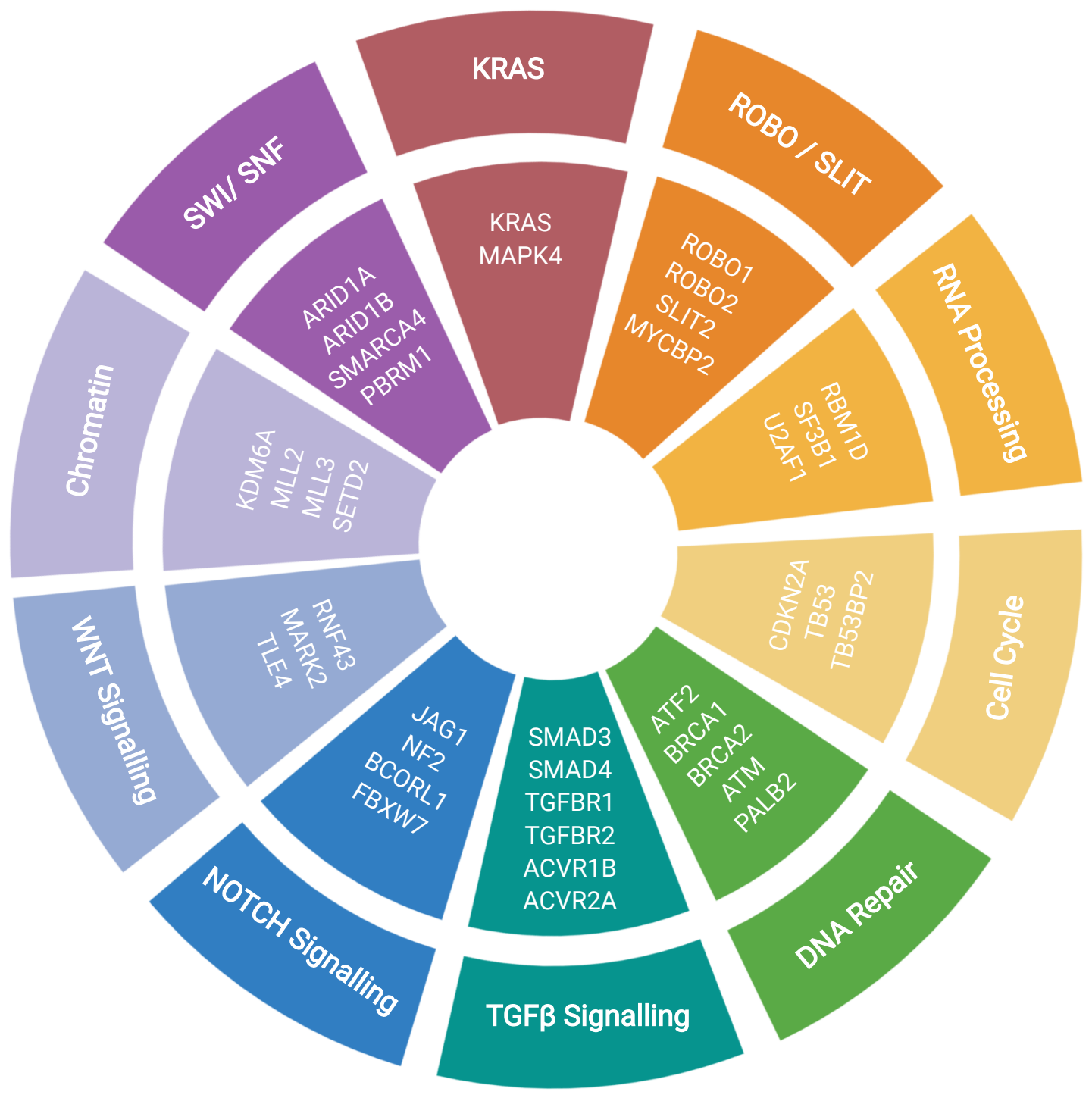




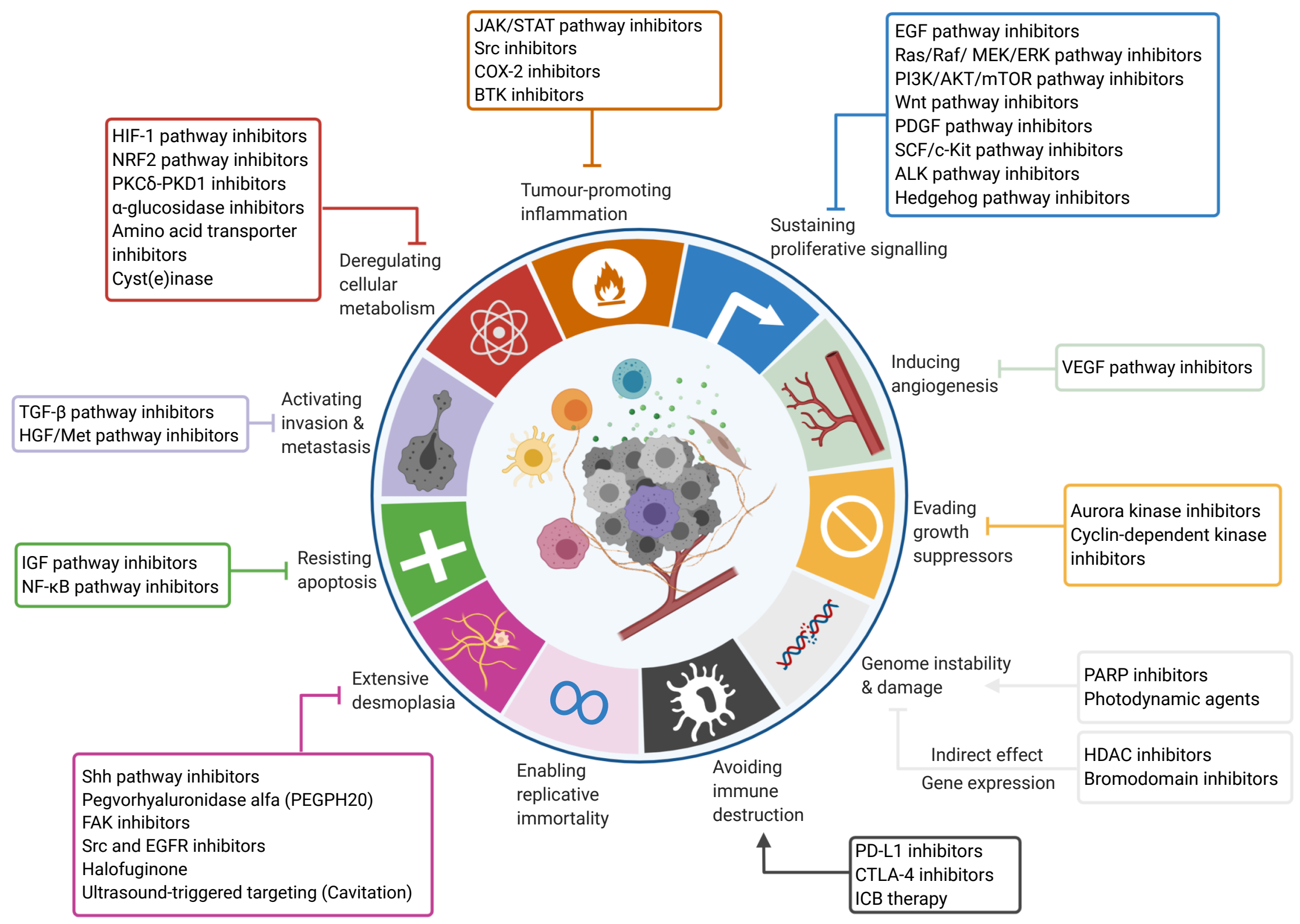



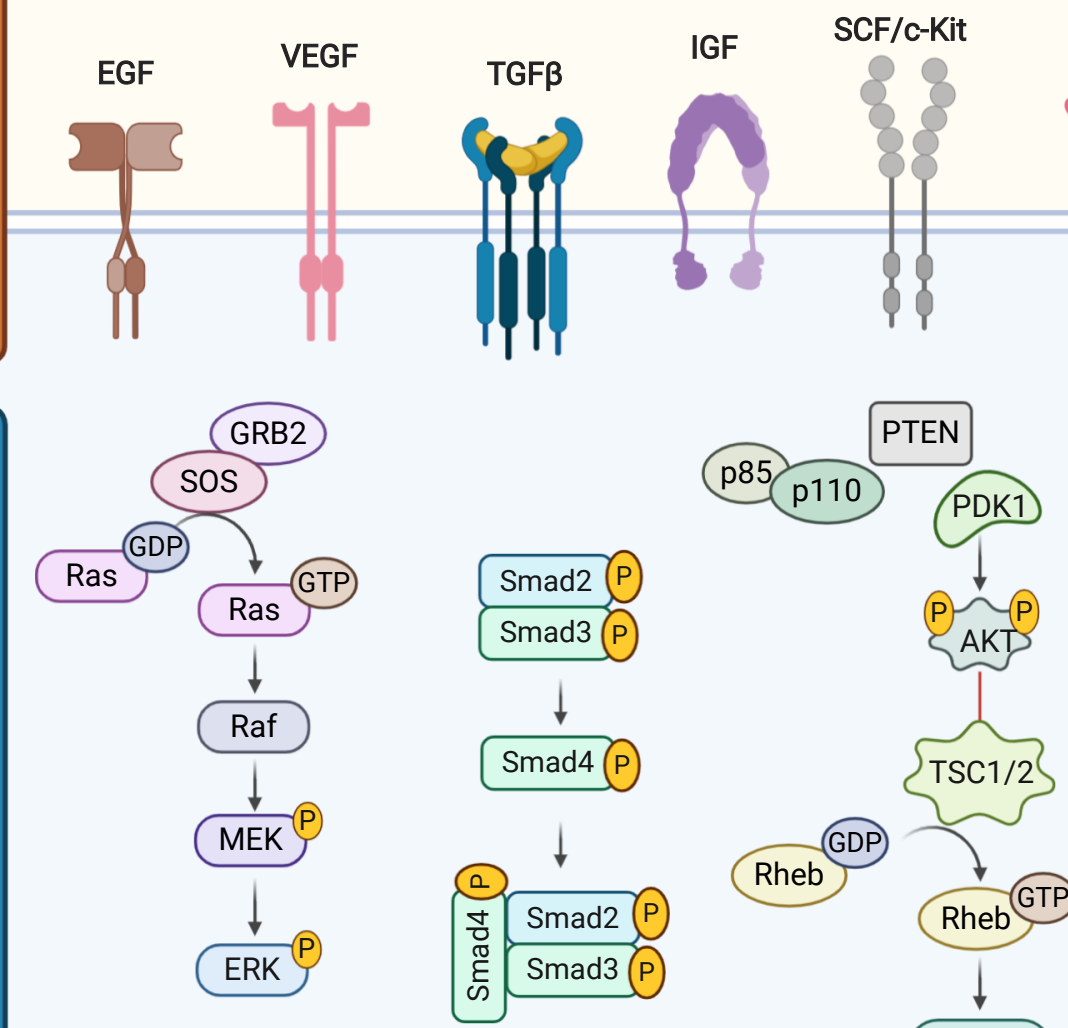

STAT3

JAKs

HGF/Met

IGF SCF/c-Kit

PDGF

2ก ALK

GDP
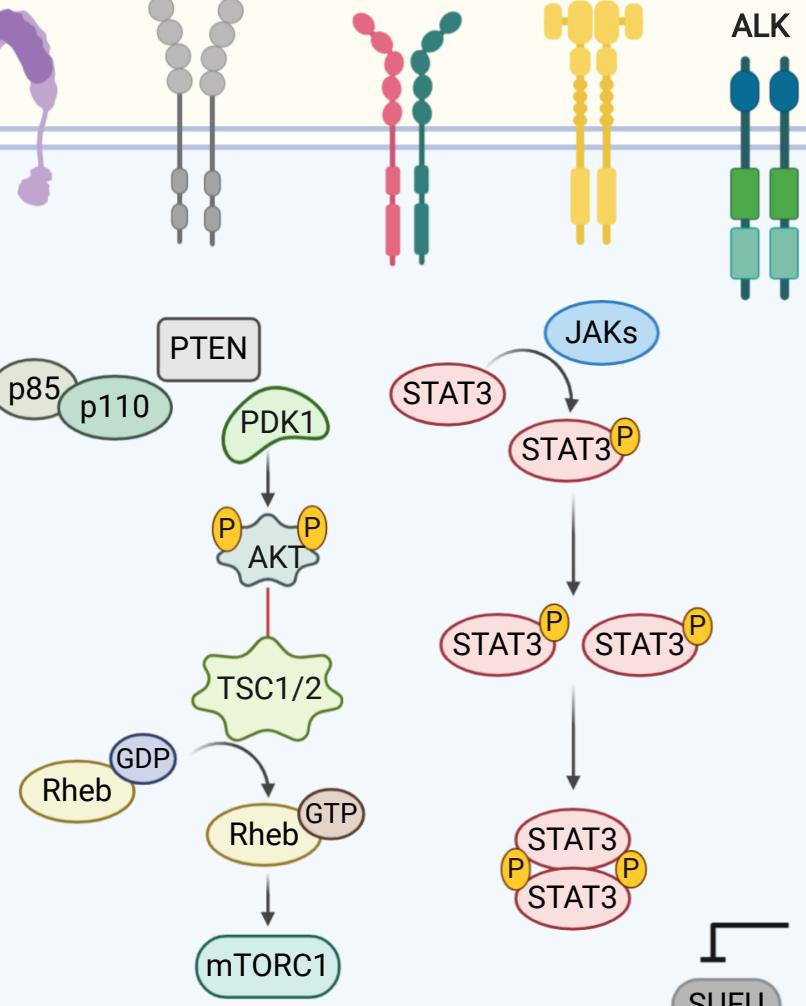

STAT3

P
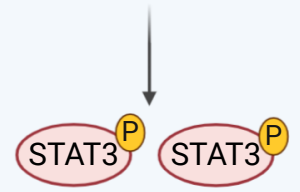

Hedgehog
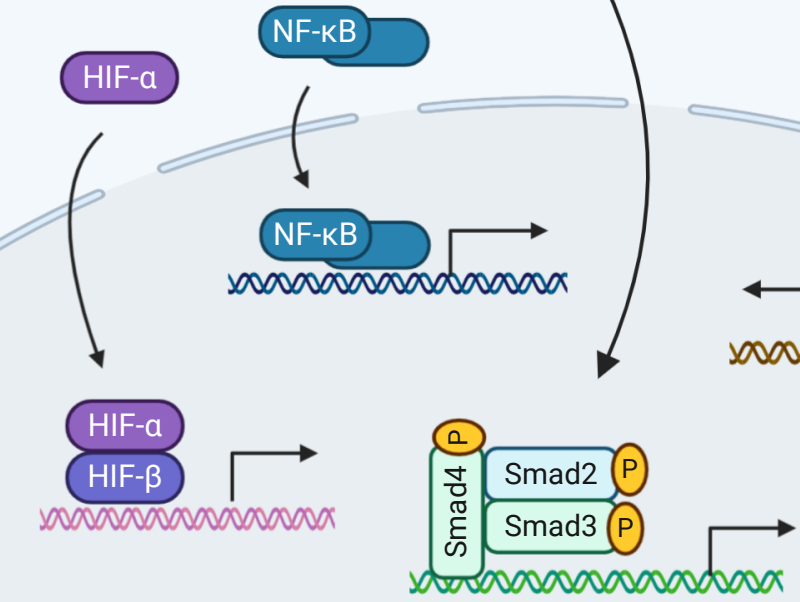

10000000000000000000
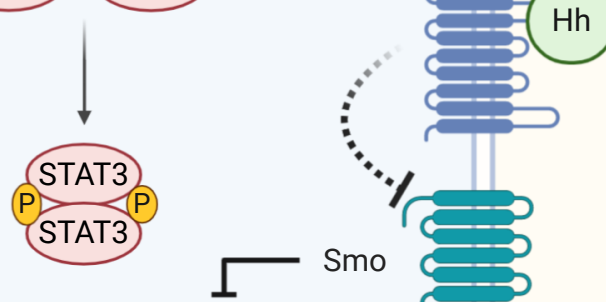

Wnt

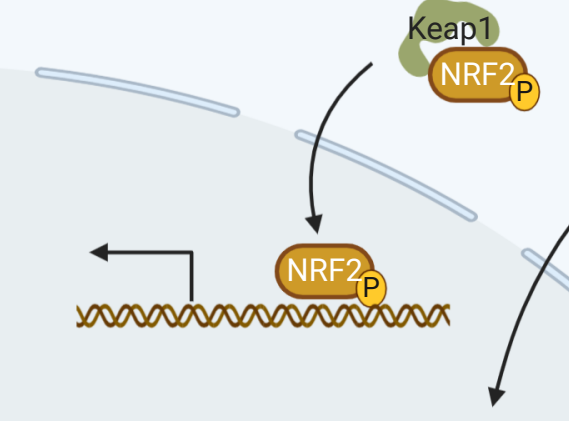

SUFU

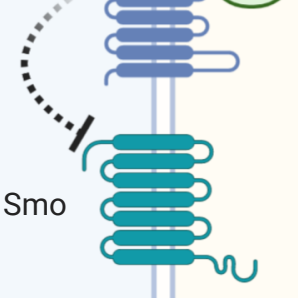

Frizzled

$\beta$-catenin

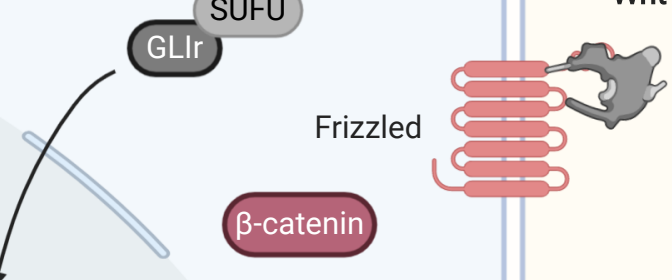

Amino acid transporter

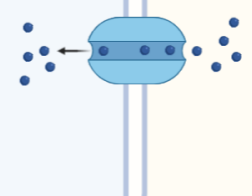


A

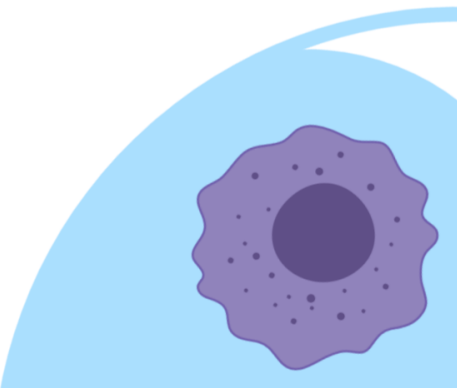

Targeting CSC

Stem cell signalling pathways Self-renewal mechanisms Cell cycle regulators Epigenetic regulators Stem cell factors
B

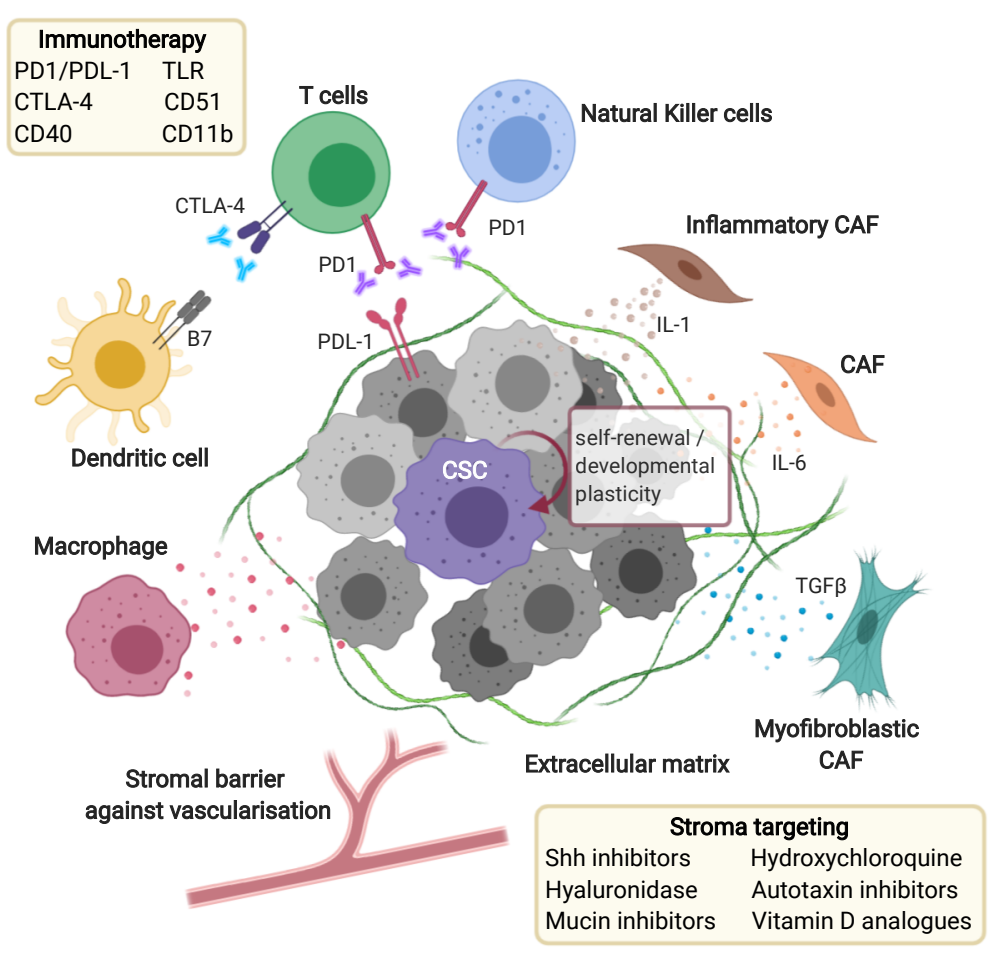

\section{Targeting non-CSC}

Antimetabolites Topoisomerases Apoptosis regulators Signalling pathways Microtubule polymerisation

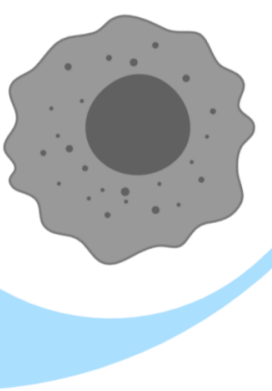




\section{Highlights}

- Pancreatic ductal adenocarcinoma (PDAC) is among the most metastatic and lethal cancers in humans.

- PDAC cells are heterogeneous and dedifferentiation leads to the formation of stem cell-like cells known as cancer stem cells (CSCs). These cells can easily metastasize are more resistant to conventional therapeutics and can lead to cancer relapse.

- As an inflammation-driven cancer, PDAC has a large stromal component that is hypovascularized, reducing access of therapeutics to cancer cells.

- Current therapeutics include FOLFIRINOX, gemcitabine, erlotinib and nab-paclitaxel, while targeted therapeutics are being explored for signalling pathways that govern the different cancer hallmarks such as proliferation, activating metastasis, metabolic effects and inflammation.

- The combined targeting of multiple features of PDAC including stroma, non-CSCs and CSCs is expected to provide improved efficiency. In particular, the targeting of the regulators of phenotypic plasticity in CSCs is an increasingly attractive research avenue. 


\section{Outstanding questions}

- Since KRAS is mutated in most PDACs with distinct hallmark mutations, can this crucial oncogenic signalling pathway be targeted in PDAC despite the lack of success in the past?

- How to overcome the physical barrier caused by the stroma and how to more efficiently deliver the therapeutics to the PDAC cells despite its hypovascularity?

- Will immunotherapy be a successful route in PDAC despite the various challenges due to the activation of signalling pathways that cause immune evasion, inflammation and desmoplasia?

- Which signalling pathways and transcription factor networks are essential for the formation and maintenance of pancreatic CSCs compared to non-CSCs?

- What are the molecular mechanisms and gene expression circuitries that provide CSCs their higher therapeutic resistance compared to other cancer cell populations?

- Which epigenetic mechanisms and stem cell factors can be targeted to synergistically disrupt cellular plasticity and to resensitize the stem cell-like cancer cells in PDAC?

- What combinations of therapeutic targets will provide the most efficient elimination of all different PDAC populations to efficiently remove the bulk tumour mass as well as CSCs that can cause metastasis and disease relapse? 